BMJ Open Sport \& Exercise Medicine

\title{
Changes in the microRNA expression profile during blood storage
}

Anna Haberberger, ${ }^{1}$ Benedikt Kirchner, ${ }^{1}$ Irmgard Riedmaier, ${ }^{1,2}$

Reinhard Henschler, ${ }^{3,4}$ Christian Wichmann, ${ }^{3}$ Raymund Buhmann, ${ }^{3}$ Michael W Pfaffl ${ }^{1}$

To cite: Haberberger A, Kirchner B, Riedmaier I, et al. Changes in the microRNA expression profile during blood storage. BMJ Open Sport \& Exercise Medicine 2018:4:e000354. doi:10.1136/

- Additional material is published online only. To view please visit the journal online (http://dx.doi.org/10.1136/10. 1136/bmjsem-2018-000354).

Accepted 27 May 2018 bmjsem-2018-000354

\section{ABSTRACT}

Objectives For several decades, autologous blood doping (ABD) in sports has been a major problem, and even today there is still no reliable method for satisfactorily detecting $A B D$. For this kind of doping, stored individual erythrocytes are used to increase stamina and endurance caused by a higher erythrocyte level in the athlete's body. Since there is growing evidence that these cells are enriched with microRNAs (miRNAs), this study has been carried out to discover and validate all miRNAs occurring in fresh blood as well as in stored blood.

Methods Therefore, small RNA Next Generation Sequencing has been performed, which allows untargeted detection of all miRNAs in a blood sample. The focus of this investigation has been to find miRNA alterations in blood bags after erythrocyte processing and during storage, as compared with fresh blood directly withdrawn from subjects. Blood samples were obtained from 12 healthy, recreationally active male subjects three times before blood donation and from blood bags at several time points after blood processing.

Results 189 miRNAs have been considered stable over two consecutive weeks. A further analysis revealed a complex biomarker signature of 28 miRNAs, consisting of 6 miRNAs that altered during 6 weeks of storage and 22 miRNAs that altered due to processing.

Conclusion These results suggest that the identified miRNA biomarker signature may be used for the detection of $A B D$. These 28 miRNA candidates are tested and verified currently in a follow-up study, a human transfusion clinical trial in healthy sportsmen.

\section{Check for updates}

${ }^{1}$ Institute of Animal Physiology and Immunology, Technical University of Munich, Freising, Germany

${ }^{2}$ Eurofins Medigenomix Forensik GmbH, Ebersberg, Germany ${ }^{3}$ Department of Transfusion Medicine, Cell Therapeutics and Hemostaseology, LudwigMaximilians-University Hospital, Munich, Germany

${ }^{4}$ Blutspende Zürich,

Medizinischer Dienst, Zurich, Switzerland

Correspondence to Michael W Pfaffl; michael. pfaff|@wzw.tum.de

\section{INTRODUCTION}

Performing homologous or autologous 'blood doping' increases the erythrocyte counts in the bloodstream of up to $10 \%$ and therefore will lead immediately to increased stamina and endurance thanks to improved oxygen-carrying capacity. After months of training, the erythrocyte mass would increase just $5 \%$.

Blood withdrawal and blood reinfusion induce changes in some haematological markers of erythropoiesis during the caused temporary anaemia and erythrocythemia. ${ }^{2}$ However, appropriate highly sensitive methods of detecting even small

\section{What are the new findings?}

RNA extracted from erythrocyte concentrates (ECs) have good RNA integrity number values appropriate for Next Generation Sequencing.

- Long-time storage of ECs resulted in an upregulation of six miRNAs (miR-16-2-3p, miR-1260a, miR1260b, miR-4443, miR-4695-3 p and miR-5100).

- A complex biomarker signature consisting of 28 miRNAs was successfully created and are currently being tested in our study.

amounts of transfused autologous blood are still missing. ${ }^{3}$ Hence new innovative methods to detect 'blood doping' and promising new prospects, for example, on the transcriptome level, ${ }^{45}$ are required in order to find new valid biomarker signatures. There are various reasons why microRNAs (miRNAs) should be taken into account when searching for new and stable biomarkers. ${ }^{6}$ miRNAs comprise a large family with more than 2500 mature miRNAs in humans, ${ }^{7}$ of evolutionarily conserved, small non-coding RNAs $(\sim 21$ nt) with great stability and long half life, ${ }^{8-10}$ which regulates gene expression by binding complementary to the 3'UTR, 5'UTR or open reading frame of target mRNA. This results either in translational inhibition or in mRNA degradation based on degree of homology. ${ }^{11}$ It is estimated that the majority (over 60\%) of human protein-coding genes are regulated by miRNAs. ${ }^{12}$ These post-transcriptional regulators play a crucial role in various biological processes, such as cell growth, tissue differentiation, cell proliferation, apoptosis ${ }^{13}$ and also haematopoiesis. ${ }^{11}$ Since several years miRNAs are also successfully used as diagnostic biomarkers for various diseases. ${ }^{14}$ It is conceivable that altered miRNA expression levels lead to disease and cellular pathologies as well as dysregulated miRNA expression is a consequence of disease. ${ }^{15}$

Since performing autologous blood doping (ABD) is associated with blood storage for several weeks, we focused on erythrocyte 
processing and storage to investigate the usefulness of miRNA in erythrocytes as a new parameter in a pilot study. We hypothesise that the non-physiological conditions of erythrocyte storage result in dysregulation in the transcriptome and in particular in a changed miRNA expression pattern as a consequence of detrimental changes in erythrocyte physiology and function, which is known as storage lesion. The aim of the study was to discover and validate all miRNAs that show alteration in blood bags during storage compared with those in fresh blood and to develop a valid biomarker signature of significant increased miRNAs, which can be used in a follow-up study with ABD.

\section{METHODS}

Subjects

Twelve healthy, recreationally active male subjects participated in the pilot study. Their anthropometric data are outlined in online supplementary table 1 . All subjects were non-smokers and free of all kinds of medication during the study course. They obtained detailed verbal and written reports about the study and signed the declaration of consent before participation. The study was performed in accordance with the latest revised form of the Declaration of Helsinki and approved by the Ethics Committee of the LMU (Ludwig-Maximilians Universität München; project no. 385-13).

\section{Study design}

To get an insight into the normal physiological baseline RNA profile of each subject, venous blood samples were taken 3, 2 and 1 week before blood donation performing standard venipuncture at the bend of the elbow using the median cubital vein. One week later, the whole blood donation (WBD) was drawn using the Composelect T3984-23 System (Fresenius, Bad Homburg, Germany) according to practice. Erythrocyte concentrates (ECs) were produced using the Compomat G4 (Fresenius, Bad Homburg, Germany) and stored at $4^{\circ} \mathrm{C}$ according to practice. EC blood samples were taken at the following points in time: 0 week, 1 week, 2 weeks, 4 weeks and 6 weeks as shown in figure 1 .

\section{Blood sampling and processing}

Venous blood samples from the first three points in time were taken directly into the PAXgene Blood RNA Tubes (PreAnalytiX, Hombrechtikon, Switzerland) using a 20-gauge butterfly needle. These tubes allow the stabilisation of the actual RNA profile including the miRNAs.

The blood donation of $500 \mathrm{~mL}$ blood was drawn into the Composelect T3984-23 System (Fresenius, Bad Homburg, Germany) containing $70 \mathrm{~mL}$ of citrate phosphate dextrose (CPD). During the blood donation, the blood was automatically mixed by a blood blending weigher (Compomixer M2, NPBI) to prevent clot formation. The blood was then leucodepleted at room temperature using the inline filter system. After a centrifugation step $\left(3998 \mathrm{~g}\right.$ for $10 \mathrm{~min}$ at $\left.20^{\circ} \mathrm{C}\right)$, the plasma was separated from the erythrocytes and most of the plasma was removed from the erythrocytes into an empty satellite bag for plasma using the Compomat G4 (Fresenius, Bad Homburg, Germany). The Compomat G4 is controlled by a computer system using the Compomaster software (Compomaster Net, V.2.01.01. D, NPBI) ${ }^{16}$ In a last step, $110 \mathrm{~mL}$ of an additive solution of phosphate, adenine, glucose, guanine, saline and mannitol was added to the erythrocytes.

For each point in time during EC storage, $18 \mathrm{~mL}$ of blood was collected from the bag for multiple blood samples. The fastest way to collect enough blood and to avoid shear forces was to clamp the hose (with a forceps) and cut it with a sterile scalpel, since RNA transcript levels change dramatically within short time intervals. ${ }^{17-19}$ To avoid air in the bag, the blood was drained from a lifted/ head first blood bag into a $50 \mathrm{~mL}$ tube. After the blood collection, the hose was immediately clamped before lowering the blood bag and welded under the forceps. Three millilitres of blood was then instantly transferred to a PAXgene Blood RNA Tube with a serological pipette

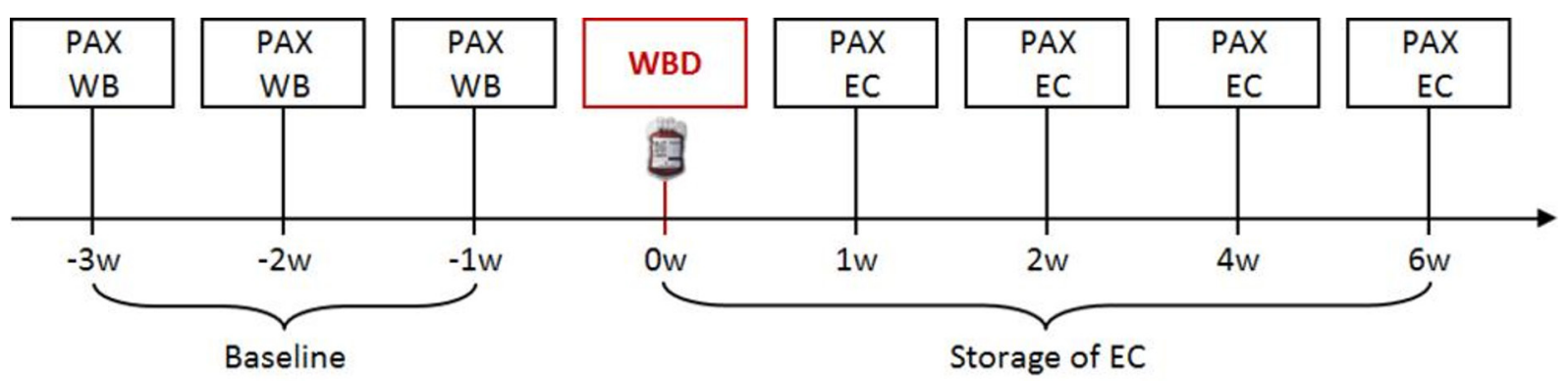

\section{Detection of the Natural \\ Variation of Gene Expression}

\section{Detection of Physiological Changes in the RNA-Profile}

Figure 1 Study design. The WB was sampled thrice before WBD and stored as $E C$ at $4^{\circ} \mathrm{C}$ afterwards for 6 weeks (w). The samples were taken at the given points in time using the PAXgene Blood RNA Tubes (PAX). EC, erythrocyte concentrate; WB, whole blood; WBD, whole blood donation. 
and gently inverted 10 times for complete lysis. ECs were stored in a $4^{\circ} \mathrm{C}$ blood bank fridge until the next sampling.

\section{Total RNA extraction}

Total RNA was extracted from PAXgene tubes using the PAXgene Blood miRNA Kit (Qiagen, Hilden, Germany) according to manufacturer's protocol until the elution step. For elution, $60 \mu \mathrm{l}$ of Buffer BR5 were pipetted directly onto the spin column and centrifuged for $1 \mathrm{~min}$ at $20000 \times \mathrm{g}$. This step was repeated with the $60 \mu \mathrm{l}$ eluate in a microcentrifuge tube. Total RNA concentration was quantified using the Qubit 2.0 Fluorometer (Life Technologies, Grand Island, New York, USA) in combination with the RNA HS Assay Kit (Life Technologies) according to the manufacturer's protocol.

To determine the RNA quality, the RNA integrity number (RIN) was measured on an Agilent 2100 (Agilent Technologies, Waldbronn, Germany) using an Agilent RNA 6000 Nano Kit (Agilent Technologies). In addition, a Small RNA Kit (Agilent Technologies) was used to measure the small RNA fraction. Both measurements were performed according to the manufacturer's protocol. ${ }^{20}{ }^{21}$ The extracted RNA was stored at $-80^{\circ} \mathrm{C}$ until further processing.

\section{Library preparation and small RNA sequencing}

For library preparation, $100 \mathrm{ng}$ of total RNA of each blood sample was converted into barcoded cDNA libraries using the NEBNext Multiplex Small RNA Library Prep Set for Illumina (New England Biolabs, Ipswich, USA). The steps of this kit include sequential adaptor ligation to both ends, reverse transcription and the introduction of unique barcodes during the PCR step to each sample. After PCR enrichment, the cDNA was purified using the MinElute PCR Purification Kit (Qiagen) and loaded on a DNA 1000 Chip (Agilent Technologies) to determine the concentration of the produced cDNA construct. For size selection, the pooled cDNA constructs were separated on a $4 \%$ agarose gel (at $4^{\circ} \mathrm{C}$ and $150 \mathrm{~V}$ for 3 hours) using MetaPhor Agarose (Lonza, Rockland, USA) for better resolution. ${ }^{22}$ The produced small RNAs are of approximately $135-160 \mathrm{bp}$ due to the adaptor ligation to both ends and the introduction of unique barcodes to each sample. To ensure that only small RNAs of interest are included in the final library, the appropriate band between 135 and $160 \mathrm{bp}$ was cut out. The cDNA from the gel slice was extracted with the MinElute Gel Extraction Kit (Qiagen) and analysed regarding size and purity on the High Sensitivity DNA Chip (Agilent Technologies). The concentration was determined with the Qubit 2.0 Fluorometer (Life Technologies) in combination with the ds HS DNA Assay Kit (Life Technologies) according to the manufacturer's protocol. The cDNA libraries were subjected to the Illumina sequencing pipeline at Next Generation Sequencing (NGS) @ TUM (NGS core facility at Technical University of Munich Weihenstephan). The pipeline consists of clonal cluster generation on a singleread flow cell (Illumina) by bridge amplification on the
cBot via TrueSeq SR Cluster Kit v3-cBOT-HS (Illumina) and 50 cycles sequencing-by-synthesis on the HiSeq2000 (Illumina).

\section{Generating a read count table}

Raw read data were processed using an in-house procedure. ${ }^{23}$ Btrim was used to trim the 3'end adapter sequence. ${ }^{24}$ Reads without detectable adapter sequence or with less than $16 \mathrm{nt}$ were excluded from the data set. The quality control software Fast QC (Babraham Bioinformatics, UK, V.0.10.1) was used to calculate sequence length distribution and representing base calling accuracy by the mean phred quality scores ( $Q$ score). For further specificity of the reads, all human rRNA, tRNA, snRNA and snoRNA sequences were removed by mapping the reads to RNAcentral database (RNAcentral Consortium 2015). For filtering, one mismatch in the first $15 \mathrm{nts}$ and a variable number of mismatches in the remaining sequence was allowed. The remaining reads were mapped to the most recent miRBase database (release 21)..$^{7}$ All alignments were carried out via Bowtie short read aligner ${ }^{25} 26$ using the 'best alignment algorithm'. The sorting and indexing of aligned reads were carried out by SAMtools ${ }^{27} 28$ and readcounts finally generated by calling the sum of hits per miRNA sequence. MiRNAs with average readcounts $<50$ were excluded from the read count table as a noise-cutoff.

\section{Statistical analysis}

All sequencing data were normalised and differential gene expression was assessed using the DeSeq2 $\mathrm{R}$ script ${ }^{29} 30$ (RStudio V.3.1.2). P values have been adjusted for multiple testing using Benjamini-Hochberg approach. Heatmap, Hierarchical Cluster Analysis and (dynamic) Principal Component Analysis were carried out with GenEx software (GenEx Pro, 5.4.4.119, Multid Analyses AB, Sweden).

\section{RESULTS}

\section{RNA quality and quantity}

The total RNA yields over all 96 samples were $4880.6 \pm 2375.6 \mathrm{ng}$ per sample, measured by fluorometric quantification. As expected, the total RNA yield was significantly different $(p<0.01)$ between the blood samples which were taken freshly versus the stored blood samples. RNA quality and RIN values were determined using the Bioanalyzer 2100 (Agilent Technologies) and resulted in integer total RNA in all samples with mean RIN values of $7.53 \pm 0.64$ (figure 2 ). The miRNA content of total RNA was $7.67 \% \pm 2.46 \%$ determined by the Bioanalyzer 2100 analysis software, with no differences between sampling groups or sampling weeks.

\section{Phred score}

For sequence accuracy, a quality value called Phred score was estimated for each sample. A mean Phred score $>30$ means a $99.9 \%$ probability of correct base call. Each sample had a mean Phred score of 37 and only $3.41 \%$ of all reads had a Phred score $<30$. 


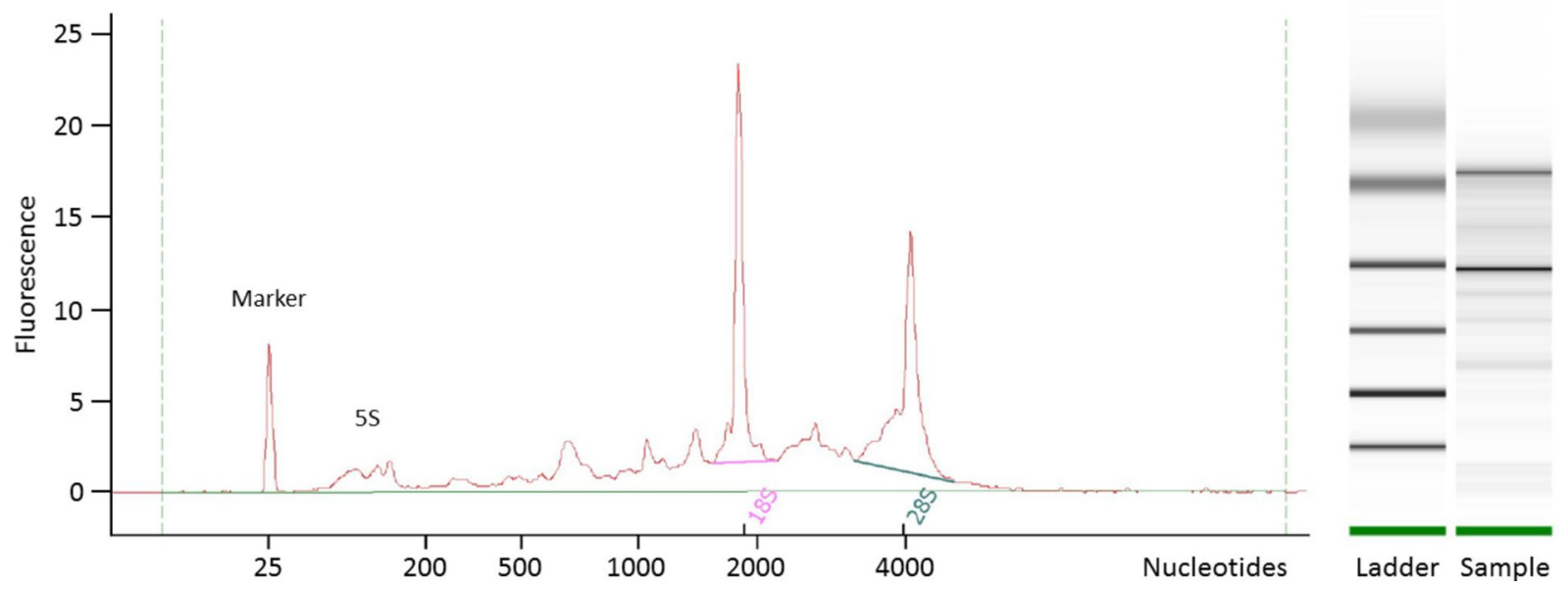

Figure 2 Electropherogram and gel image to calculate RNA quality. A typical electropherogram of total RNA of human EC. This example shows a sample with good quality (RIN: 7.4). EC, erythrocyte concentrate; RIN, RNA integrity number.

\section{Data evaluation}

Raw reads per sample with an average of $12.0 \pm 5.45 \times 10^{6}$ were obtained. Based on a shorter read length than 16 nucleotides $4.51 \pm 2.96 \times 10^{6}$ reads per sample were excluded. The remaining $7.52 \pm 3.35 \times 10^{6}$ reads were mapped to the RNAcentral database to remove all human rRNAs, tRNAs, snRNAs and snoRNAs. After excluding $2.60 \pm 1.51 \times 10^{6}$ reads mapped to RNAcentral, the remaining $2.69 \pm 2.29 \times 10^{6}$ reads were mapped to 2588 annotated human miRNAs collected in the miRBase database. $2.99 \pm 1.35 \times 10^{6}$ reads aligned to 1734 different human miRNAs which correspond to $32.4 \%$ of the raw reads.

To avoid background noise and false positive, an average threshold of $\geq 50$ reads over all samples was set. This excluded 1539 miRNAs and 195 human miRNAs were considered for further analysis. Most of these miRNAs (113) have the typical miRNA sequence length of 22 nucleotides, which corresponds to exactly $90.0 \%$ of all remaining reads. More information about the abundance of miRNAs and their corresponding sequence length is shown in online supplementary table 2 and figure 1 .

\section{Natural quantity variation in miRNA expression}

To identify natural quantity variations in miRNA expression, three sampling time points were taken over 2 weeks. Between two following samplings, that is, during 1 week, there were no significant differences (3 weeks before vs 2 weeks before and 2 weeks before vs 1 week before). During three samplings over 2 following weeks, small differences occurred only in six miRNAs out of 195 (online supplementary table 3 ). Between time points $3 \mathrm{w}$ before and $1 \mathrm{w}$ before let-7g-5p, miR-126-3 p, let-7a-5p, let-7i-5p, miR-107 and miR-107 were downregulated.
Based on this significant expression changes $(p<0.05)$, these miRNAs were excluded from further analysis.

\section{Most significantly upregulated miRNAs after blood bag processing}

For ABD, stored blood bags must be transfused to the donor to increase their erythrocytes. In order to reveal doping sinners on the basis of a different miRNA expression profile only highly upregulated miRNAs, which increase their normal miRNA level, can be used. Since three samples were taken on a weekly basis to identify natural variation, every single one of these time points were compared with time point $0 \mathrm{w}$, the sample directly taken after processing the blood bag. This resulted in three different tables where the miRNAs were sorted according to the smallest $p$ value. After excluding the unstable miRNAs and the downregulated miRNAs, the Top 20 significantly regulated miRNAs of time point 0 $\mathrm{w}$ of each table were combined into one table with 22 miRNAs (table 1). Almost every miRNA in the initially Top $200 \mathrm{w}$ could be found in all three tables. Only two miRNAs were different ones. To set up only one table the Top $200 \mathrm{w}$ miRNAs were expanded to a Top 220 $\mathrm{w}$ miRNA list with the missing miRNAs from the other tables. In the further course of the paper, this Top $220 \mathrm{w}$ list with the greatest $p$ value will be referred to as Top 22 $0 \mathrm{w} p v$ ( $\mathrm{p}$ value). The Top $220 \mathrm{w}$ pv are ranked for each time point before blood donation. MiRNAs that did nOt occur in every table are marked with bold rank numbers.

\section{Most affected miRNAs by blood bag processing}

A list with the Top $220 \mathrm{w}$ miRNAs with the greatest fold change (Top $220 \mathrm{w} \mathrm{fd}$ ) was created as described above. The miRNAs were sorted according to the greatest fold change in expression, the three tables were combined 
Table 1 Top 22 upregulated miRNAs with the greatest $p$ values

\begin{tabular}{|c|c|c|c|c|c|c|c|c|c|}
\hline \multirow[b]{2}{*}{ miRNA } & \multicolumn{3}{|c|}{$3 \mathrm{w}$ before vs $0 \mathrm{w}$} & \multicolumn{3}{|c|}{$2 \mathrm{w}$ before vs $0 \mathrm{w}$} & \multicolumn{3}{|c|}{$1 \mathrm{w}$ before vs $0 \mathrm{w}$} \\
\hline & Rank & $\begin{array}{l}\text { Fold } \\
\text { change }\end{array}$ & $P$ values & Rank & fc & P values & Rank & fc & P values \\
\hline miR-20a-5p & 2 & 11.92 & $1.41 \mathrm{E}-27$ & 1 & 16.63 & $3.51 \mathrm{E}-35$ & 2 & 19.66 & $2.58 \mathrm{E}-39$ \\
\hline miR-454-3p & 3 & 9.68 & 2.97E-26 & 3 & 12.74 & $4.42 E-32$ & 3 & 18.16 & 6.64E-39 \\
\hline miR-93-5 p & 6 & 4.96 & 1.62E-22 & 6 & 6.14 & $8.21 \mathrm{E}-29$ & 7 & 6.63 & $3.14 \mathrm{E}-31$ \\
\hline miR-27b-3p & 7 & 3.74 & $1.84 \mathrm{E}-21$ & 5 & 5.03 & $1.79 \mathrm{E}-31$ & 4 & 5.61 & $2.86 \mathrm{E}-35$ \\
\hline miR-20b-5p & 8 & 7.13 & $1.71 \mathrm{E}-20$ & 7 & 9.77 & 4.79E-27 & 8 & 10.16 & 8.03E-28 \\
\hline let- $7 b-5 p$ & 9 & 3.68 & 3.63E-20 & 12 & 3.68 & 2.73E-20 & 9 & 4.55 & 5.83E-27 \\
\hline miR-106a-5p & 13 & 5.31 & 1.10E-15 & 9 & 7.35 & 8.73E-22 & 15 & 7.66 & 1.32E-22 \\
\hline$m i R-15 b-5 p$ & 14 & 3.70 & 1.19E-14 & 11 & 4.86 & $6.18 \mathrm{E}-21$ & 11 & 5.64 & 9.97E-25 \\
\hline $\operatorname{miR}-15 a-5 p$ & 15 & 6.93 & $1.38 \mathrm{E}-13$ & 16 & 9.61 & $4.32 \mathrm{E}-18$ & 16 & 11.98 & 3.05E-21 \\
\hline $\operatorname{miR}-21-5 p$ & 16 & 5.07 & $1.45 \mathrm{E}-13$ & 15 & 6.72 & $2.52 \mathrm{E}-18$ & 13 & 8.92 & 9.92E-24 \\
\hline miR-18a-5p & 17 & 4.55 & $8.22 \mathrm{E}-13$ & 17 & 6.32 & $2.40 \mathrm{E}-17$ & 17 & 7.51 & 5.97E-19 \\
\hline miR-574-5 p & 18 & 3.23 & 2.92E-12 & 21 & 3.02 & $3.41 \mathrm{E}-11$ & 22 & 3.70 & $2.15 \mathrm{E}-14$ \\
\hline $\operatorname{miR}-144-3 p$ & 19 & 9.32 & 5.13E-12 & 19 & 10.36 & $4.72 \mathrm{E}-13$ & 18 & 15.66 & 6.37E-17 \\
\hline miR-103a-3p & 20 & 2.92 & 1.32E-09 & 18 & 3.57 & $3.84 \mathrm{E}-13$ & 21 & 3.89 & $6.79 \mathrm{E}-15$ \\
\hline
\end{tabular}

miRNAs that are not listed in every Top 20 pv are marked with bold rank numbers. miRNAs that are missing in the Top $220 \mathrm{w} f \mathrm{c}$ are underlined.

and expanded to include two miRNAs to generate one single table. Again miRNAs that did not occur in every table are marked with bold rank numbers. 19 miRNAs out of the Top $220 \mathrm{w}$ fd were identical with the Top $220 \mathrm{w}$ pv. Only three miRNAs (miR-574-5 p, -103a-3p, $-106 b-5 p /$ let-7f-5p, miR-27a-3p, -101-3 p) were different ones, which refer to the underlined miRNAs in table 1 and online supplementary table 4.

\section{Changes during blood bag storage}

During blood storage miR-16-2-3p, miR-1260a, miR-1260b, miR-4443, miR-4695-3 p and miR-5100 were found to be significantly upregulated over time (table 2 ).

None of these miRNAs were part of unstable miRNAs, Top 22 p value or Top 22-fold change lists as shown in table 1 and online supplementary table 4 .

\section{Most affected miRNAs at time point $\mathbf{6}$ w}

It is known that the body needs several weeks to replenish harvested cells and to normalise blood variables like iron and $\mathrm{Hb}^{31}{ }^{32}$ So it can be assumed that doping sinners will wait for at least 6 weeks before transfusion of the own blood bag. Therefore, the differences between the time points before and time point $6 \mathrm{w}$ are important to identify the most affected miRNA due to blood bag processing and storage. Likewise every time point before the blood donation was compared with time point $6 \mathrm{w}$ and a common list of Top $216 \mathrm{w}$ pv was generated base on $p$ values (table 3 ). The same procedure was performed with the lists based on fold changes to generate a list of Top $226 \mathrm{w}$ fc (online supplementary table 5). Tables 2 and 3 and online supplementary tables 4 and 5 did not differ much from each other. The only new miRNAs were the ones that changed during blood bag storage, like miR-1260a, miR-1260b, miR-4695-3 p and miR-5100.

\section{Multivariate analysis}

The Top $220 \mathrm{w}$ pv and the Top $216 \mathrm{w}$ pv from time point $1 \mathrm{w}$ before were analysed by PCA (figure $3 \mathrm{~A}$ ). The first component (PC1) explained $79.7 \%$ and $74.6 \%$ of the total variance, respectively. Based on the selected miRNAs, two clear clusters formed between samples taken from subjects (Arm) and those taken from stored blood bags (Bag). The corresponding heatmaps showed the same picture of well separated samples from arm (red bars) and blood bags (blue bars) (figure 3B) . 


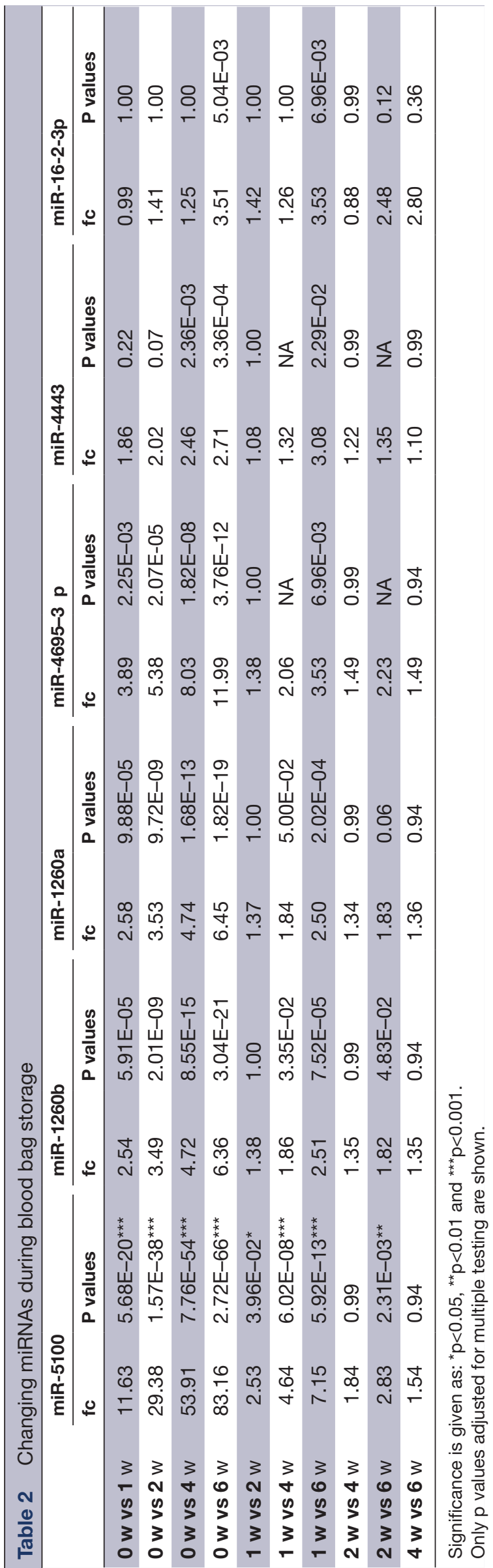

\section{DISCUSSION}

\section{Huge amount of RNA in erythrocytes}

For a long time, erythrocytes have been thought to lack genetic material as compared with their progenitors, the reticulocytes. ${ }^{33}$ For a decade now, there is growing evidence that erythrocytes contain plenty miRNAs, ${ }^{34} 35$ even more than in other blood fractions. ${ }^{36}$ Hence, we focused our biomarker discovery on the most promising RNA family, the miRNAs. Final goal is the establishment of a stable and valid biomarker signature to detect $\mathrm{ABD}$. In this study, erythrocytes were obtained from ECs and not from density gradients. Although, compared with whole blood, the percentage of erythrocytes in blood bag units, measured using the haematocrit value, is between $50 \%$ and $70 \%,{ }^{37}$ because WBCs and platelets were filtered out. Most of the plasma and the conservation buffer CPD were removed down to a minimum of a few millilitres. Hence, it can be assumed that free plasma RNA does not make a significant contribution to the extracted total RNA and that the total RNA were gained mainly from erythrocytes. This study should further show that erythrocytes in fact contain a huge amount of total RNA including diverse miRNAs and typical eukaryotic 5S, $18 \mathrm{~S}$ and 28S rRNA subfractions. ${ }^{38}$ These rRNA subfractions can be used in addition to calculate the RNA quality based on a Bioanalyzer RIN analysis, in order to use only biological blood samples with high RIN values for further analysis, whereas Chen et al could not find any rRNA subfractions. ${ }^{35}$

\section{Unsuitable miRNAs as biomarker}

After the successful RNA extraction from ECs, it was important to determine the natural variation of all miRNAs in healthy donors. Over a time period of 2 weeks, six 'unstable miRNAs' (eg, let-7g-5p) were identified and excluded from further analysis. Leuenberger et al, who investigated circulating miRNAs as biomarkers for ABD, discovered let- $7 \mathrm{~g}$ being one of the most affected circulating miRNA after blood transfusion. ${ }^{39}$ Together with our finding, it would not be advisable to use this miRNA as a biomarker, since it shows large natural arbitrary variation in gene expression.

\section{Dilution factor of upregulated miRNAs}

Since erythrocytes contain plenty of miRNAs and ABD is based on transfusion of ECs to increase the erythrocyte count in the body, this transfusion should also lead to a couple of increased miRNAs in the EC. Clearly, not all of the so far identified upregulated and increased miRNAs can be found in the body after transfusion due to dilution. A small blood bag of approximately $280 \mathrm{~mL}^{39}$ dilutes in the body with approximately $5.43 \mathrm{~L}$ of blood after Nadler's formula for men. ${ }^{29}$

According to this formula, the dilution factor would be around 20 for one EC or only around 10 for two ECs. Only highly increased miRNAs will lead to a measurable difference in the miRNA profile of a treated subject. Although it is not only conceivable that miRNAs from the EC will increase the level of the miRNA in the body, it is 
Table 3 Top 21 upregulated miRNAs with the greatest $p$ values

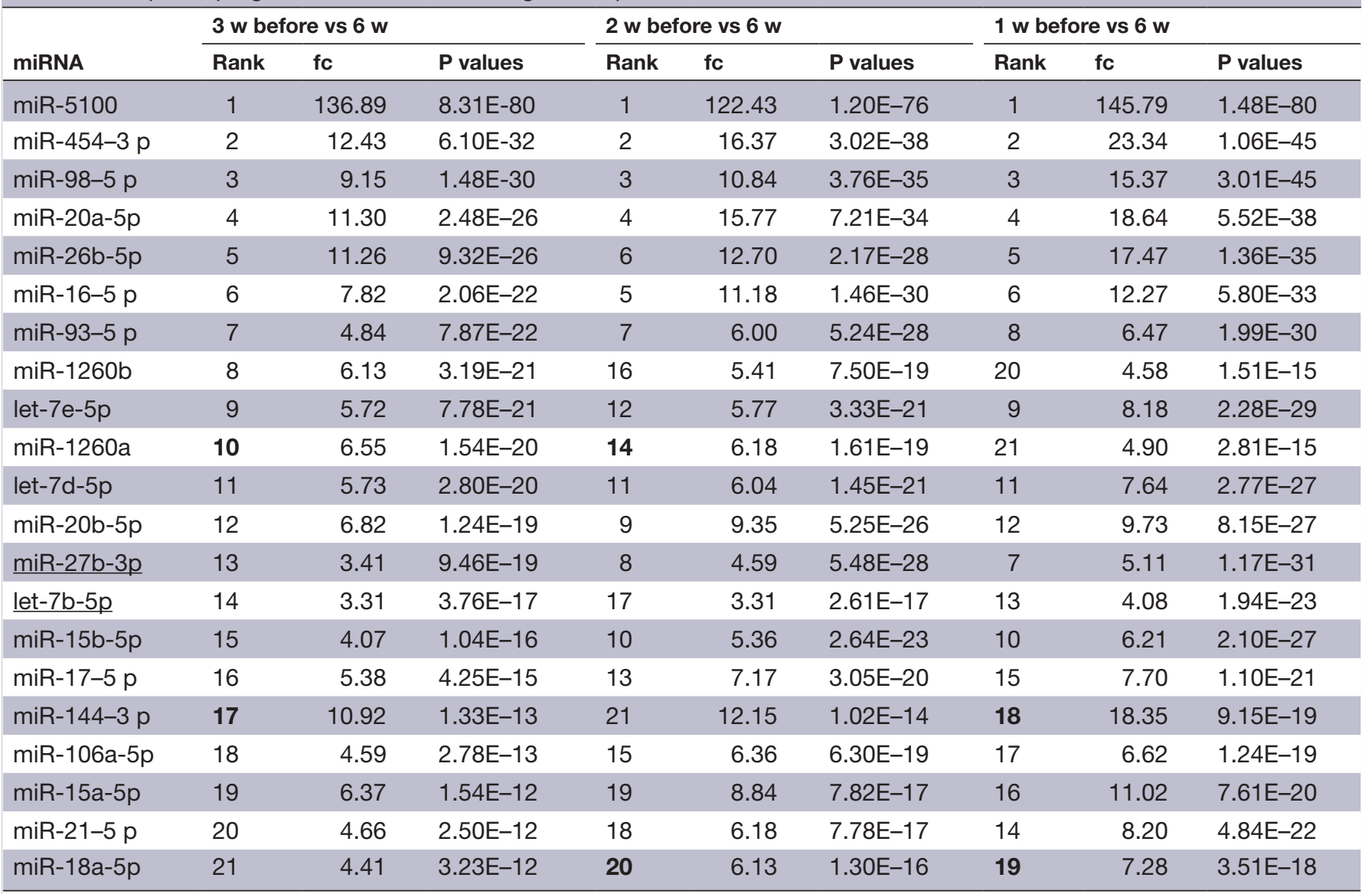

miRNAs that are not listed in every Top $206 \mathrm{w}$ pv are marked with bold rank numbers. miRNAs that are missing in the Top $226 \mathrm{w}$ fc are underlined.

also possible that erythrocyte and other cells, which have their origin in the body, respond to the blood or erythrocyte donation. Either the storage buffer, the increased erythrocyte count and/or the transfusion stress can change the miRNA gene expression.

\section{Haemolysis}

Several studies have shown that miRNAs in circulation are affected by haemolysis. ${ }^{27} 364041$ Ruptured erythrocytes release their content into the plasma, which, depending on the haemolysis level, leads to a different miRNA signature. ${ }^{27}$ Therefore, haemolysed samples should be discarded out when searching for reliable miRNA biomarkers in plasma or serum. In this case, miRNAs have not been detected in cell-free plasma or serum but in whole blood and ECs where all cells were lysed. Hence, the haemolysis level should not have an impact on the miRNA profile when total RNA extraction is done from lysed samples.

\section{Complex biomarker signature needed}

The major goal of this study was to create a potential 'biomarker signature' of miRNAs in ECs in order to detect ABD. Therefore, miRNAs were selected according to their highly increased expression during storage and they should distinguish between fresh and stored blood samples. For creating such a 'biomarker signature', various parameters like timepoints, $p$ value and foldchange were taken into account (figure $3 \mathrm{~A}$ and $\mathrm{B}$ ).

The use of a single biomarker would limit the specificity because of the numerous and complex underlying mechanisms of an autologous blood transfusion. Compared with the use of a complex biomarker signature, the likelihood of identifying doping sinners is strongly improved. A complex signature reduces false positive as well as false negative results.

In a follow-up study, the practicality and applicability of these biomarker signatures are currently being tested including actual ABD. The most promising single miRNA candidate was miR-5100. With an increase of over 120 -fold change, there should not be a problem to detect this increase in the body after transfusion and is expected to be part of the final biomarker signature.

\section{CONCLUSION}

In conclusion, erythrocytes contain a huge amount of total RNA with a high miRNA fraction. The miRNA expression of 198 miRNAs was stable over two consecutive weeks, except for six miRNAs for creating the biomarker signature. After blood bag processing, consisting of filtration, addition of preservative solution and centrifugation, 
A

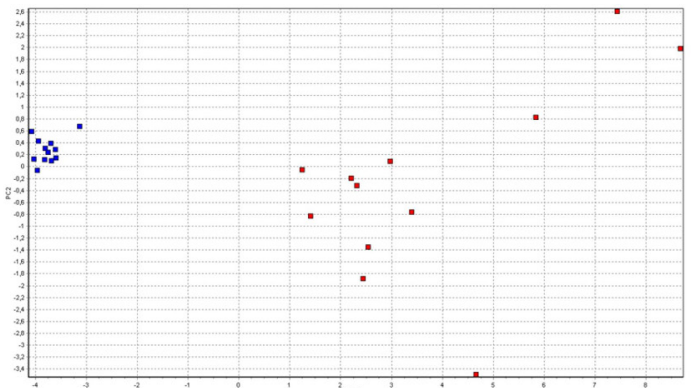

B

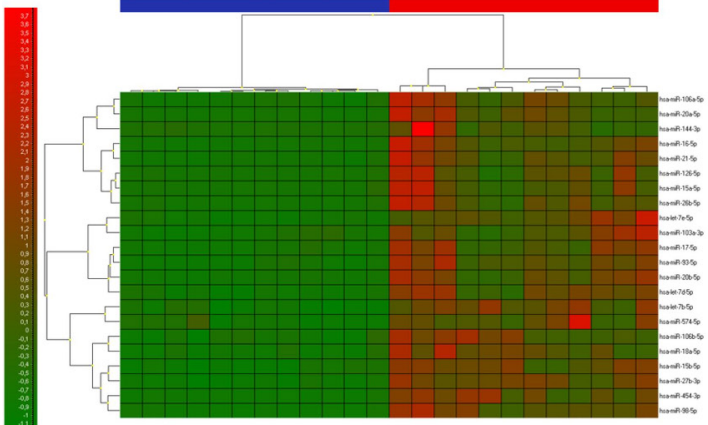

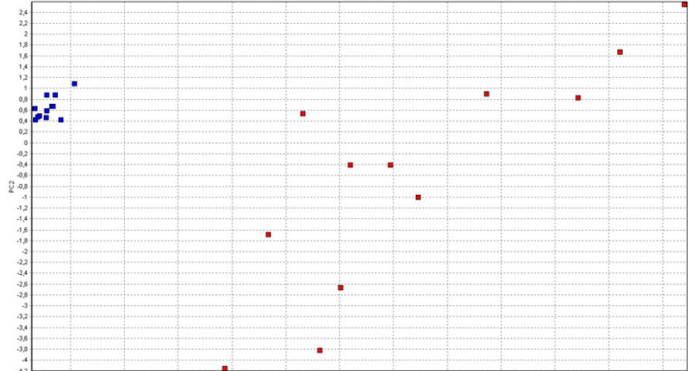

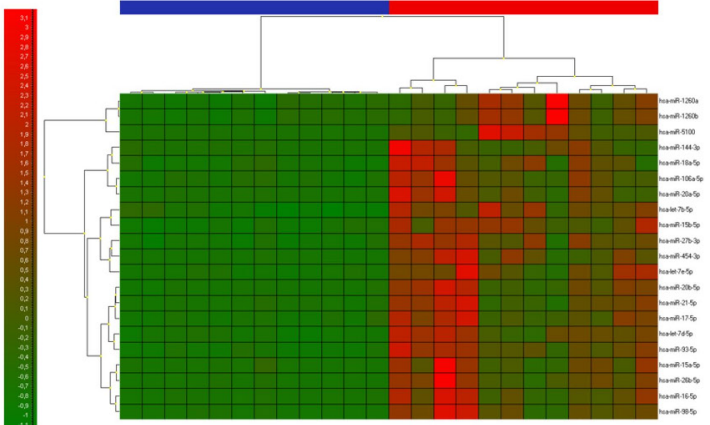

Figure 3 Multivariate analysis. (A) Red and blue indicate samples from a blood bag and from a patient's arm, respectively. On the left side are the Top $220 \mathrm{w}$ pv loaded with the samples from time points $1 \mathrm{w}$ before vs $0 \mathrm{w}$. On the right side are the Top $216 \mathrm{w}$ pv loaded with the samples from time points $1 \mathrm{w}$ before vs $6 \mathrm{w}$. The first component (PC1) explained $79.7 \%$ and $74.6 \%$ of the total variance, respectively. (B) The associated heatmaps are shown directly below the PCAs. Red and blue indicate bars represent samples from a blood bag and from a patient's arm, respectively. Green and red colours in heatmap represent low and high expression of the miRNAs, respectively. More details about the miRNAs used for the heatmaps can be found in tables, respectively.

ECs revealed a highly different miRNA expression profile compared with fresh drawn blood. In addition, storage over a period of 6 weeks had an impact on six miRNAs. The biomarker search resulted in 28 differential regulated miRNAs after considering timepoints, $p$ values and fold changes. The identified 28 miRNAs are the most promising ones for a prospective biomarker signature to detect ABD. These 28 miRNA candidates are tested and verified currently in a follow-up study, a human transfusion clinical trial in healthy sportsmen.

Acknowledgements The authors acknowledge Dr med. Georg Wittmann from the Department of Transfusion Medicine, Cell Therapeutics and Hemostaseology, Ludwig-Maximilians-University Hospital, Munich, Germany for supporting the study.

Contributors AH, IR and MWP performed the conception and design of the study. $\mathrm{AH}, \mathrm{CW}$ and $\mathrm{RB}$ were responsible for sampling. $\mathrm{AH}$ and $\mathrm{BK}$ performed data analysis. $\mathrm{BK}, \mathrm{IR}, \mathrm{RH}$ and MWP reviewed and revised the paper.

Funding The study was funded by the Federal Institute of Sports Science (Bundesinstitut für Sportwissenschaft, BiSp: AZ 070301/13).

Competing interests None declared.

Patient consent Not required.

Ethics approval LMU Ethics Committee-Ludwig-Maximilians Universität München; project no. 385-13.

Provenance and peer review Not commissioned; internally peer reviewed.

Open access This is an Open Access article distributed in accordance with the Creative Commons Attribution Non Commercial (CC BY-NC 4.0) license, which permits others to distribute, remix, adapt, build upon this work non-commercially, and license their derivative works on different terms, provided the original work is properly cited and the use is non-commercial. See: http://creativecommons.org/ licenses/by-nc/4.0/ (c) Article author(s) (or their employer(s) unless otherwise stated in the text of the article) 2018. All rights reserved. No commercial use is permitted unless otherwise expressly granted.

\section{REFERENCES}

1. Leigh-Smith S. Blood boosting. Br J Sports Med 2004;38:99-101.

2. Damsgaard R, Munch T, Mørkeberg J, et al. Effects of blood withdrawal and reinfusion on biomarkers of erythropoiesis in humans: Implications for anti-doping strategies. Haematologica 2006;91:1006-8.

3. D'Alessandro A, Liumbruno G, Grazzini G, et al. Red blood cell storage: the story so far. Blood Transfus 2010;8:82-8.

4. Pitsiladis YP, Durussel J, Rabin O. An integrative 'omics' solution to the detection of recombinant human erythropoietin and blood doping. Br J Sports Med 2014;48:856-61.

5. Riedmaier I, Benes V, Blake J, et al. RNA-sequencing as useful screening tool in the combat against the misuse of anabolic agents. Anal Chem 2012;84:6863-8.

6. Riedmaier I, Pfaffl MW. Transcriptional biomarkers--high throughput screening, quantitative verification, and bioinformatical validation methods. Methods 2013;59:3-9.

7. Kozomara A, Griffiths-Jones S. miRBase: annotating high confidence microRNAs using deep sequencing data. Nucleic Acids Res 2014;42(Database issue):D68-D73.

8. Becker C, Hammerle-Fickinger A, Riedmaier I, et al. mRNA and microRNA quality control for RT-qPCR analysis. Methods 2010;50:237-43.

9. Winter J, Diederichs S. Argonaute proteins regulate microRNA stability: Increased microRNA abundance by Argonaute proteins is due to microRNA stabilization. RNA Biol 2011;8:1149-57.

10. Lv YH, Ma KJ, Zhang $\mathrm{H}$, et al. A time course study demonstrating mRNA, microRNA, 18S rRNA, and U6 snRNA changes to estimate PMI in deceased rat's spleen. J Forensic Sci 2014;59:1286-94.

11. Undi RB, Kandi R, Gutti RK. MicroRNAs as Haematopoiesis Regulators. Adv Hematol 2013;2013:695754-.

12. Friedman RC, Farh KK, Burge CB, et al. Most mammalian mRNAs are conserved targets of microRNAs. Genome Res 2009;19:92-105. 
13. Esquela-Kerscher A, Slack FJ. Oncomirs - microRNAs with a role in cancer. Nat Rev Cancer 2006;6:259-69.

14. Lu M, Zhang Q, Deng M, et al. An analysis of human microRNA and disease associations. PLoS One 2008;3:e3420.

15. Ryan P, Atreya C. Blood cell microRNAs: what are they and what future do they hold? Transfus Med Rev 2011;25:247-51.

16. van der Meer P, Pietersz R, Hinloopen B, et al. Automated separation of whole blood in top and bottom bags into components using the Compomat G4. Vox Sang 1999;76:90-9.

17. Rainen L, Oelmueller U, Jurgensen $\mathrm{S}$, et al. Stabilization of mRNA expression in whole blood samples. Clin Chem 2002;48:1883-90.

18. Tanner MA, Berk LS, Felten DL, et al. Substantial changes in gene expression level due to the storage temperature and storage duration of human whole blood. Clin Lab Haematol 2002;24:337-41.

19. PreAnalytiX, PAXgene Blood RNA. 2010. http://www.preanalytix. $\mathrm{com} / \mathrm{sites} /$ default/files/brochures/PAXgene\%20Blood\%20RNA\% 20System\%20Brochure.pdf (accessed 11 Jan 2017).

20. Technologies A. Agilent RNA 6000 Nano Kit Guide. 2013. http:// www.agilent.com/cs/library/usermanuals/Public/G2938-90034_ RNA6000Nano_KG.pdf (accessed 11 Jan 2017).

21. Technologies A. Agilent Small RNA. 2013. http://www.agilent.com/ cs/library/usermanuals/Public/G2938-90093_SmallRNA_KG_EN.pdf (accessed 11 Jan 2017).

22. Buschmann D, Haberberger A, Kirchner B, et al. Toward reliable biomarker signatures in the age of liquid biopsies - how to standardize the small RNA-Seq workflow. Nucleic Acids Res 2016;44:5995-6018.

23. Spornraft M, Kirchner B, Haase B, et al. Optimization of extraction of circulating RNAs from plasma--enabling small RNA sequencing. PLoS One 2014;9:e107259.

24. Kong Y. Btrim: a fast, lightweight adapter and quality trimming program for next-generation sequencing technologies. Genomics 2011;98:152-3.

25. Mørkeberg J. Detection of autologous blood transfusions in athletes: a historical perspective. Transfus Med Rev 2012;26:199-208.

26. Langmead B, Trapnell C, Pop M, et al. Ultrafast and memoryefficient alignment of short DNA sequences to the human genome. Genome Biol 2009;10:R25.

27. Kirschner MB, Edelman JJ, Kao SC, et al. The Impact of Hemolysis on Cell-Free microRNA Biomarkers. Front Genet 2013:4:94.
28. Li H, Handsaker B, Wysoker A, et al. The Sequence Alignment/Map format and SAMtools. Bioinformatics 2009;25:2078-9.

29. Nadler SB, Hidalgo JH, Bloch T. Prediction of blood volume in normal human adults. Surgery 1962;51:224-32.

30. Love MI, Huber W, Anders S. Moderated estimation of fold change and dispersion for RNA-seq data with DESeq2. Genome Biol 2014;15:550.

31. Ziegler AK, Grand J, Stangerup I, et al. Time course for the recovery of physical performance, blood hemoglobin, and ferritin content after blood donation. Transfusion 2015;55:898-905.

32. Schotten N, Pasker-de Jong PC, Moretti D, et al. The donation interval of 56 days requires extension to 180 days for whole blood donors to recover from changes in iron metabolism. Blood 2016;128:2185-8.

33. Hamilton AJ. MicroRNA in erythrocytes. Biochem Soc Trans 2010;38(Pt 1):229-31.

34. Rathjen T, Nicol C, McConkey G, et al. Analysis of short RNAs in the malaria parasite and its red blood cell host. FEBS Lett 2006;580:5185-8.

35. Chen SY, Wang Y, Telen MJ, et al. The genomic analysis of erythrocyte microRNA expression in sickle cell diseases. PLoS One 2008;3:e2360.

36. Kirschner MB, Kao SC, Edelman JJ, et al. Haemolysis during sample preparation alters microRNA content of plasma. PLoS One 2011;6:e24145

37. Liumbruno G, Bennardello F, Lattanzio A, et al. Recommendations for the transfusion of red blood cells. Blood Transfus 2009;7:49-64.

38. Kabanova S, Kleinbongard P, Volkmer J, et al. Gene expression analysis of human red blood cells. Int J Med Sci 2009;6:156-9.

39. Leuenberger N, Schumacher YO, Pradervand S, et al. Circulating microRNAs as biomarkers for detection of autologous blood transfusion. PLoS One 2013;8:e66309.

40. Pritchard CC, Kroh E, Wood B, et al. Blood cell origin of circulating microRNAs: a cautionary note for cancer biomarker studies. Cancer Prev Res 2012;5:492-7.

41. McDonald JS, Milosevic D, Reddi HV, et al. Analysis of circulating microRNA: preanalytical and analytical challenges. Clin Chem 2011;57:833-40. 